GHANA JOURNAL OF DEPARTMENT OF HEALTH, PHYSICAL EDUCATION AND RECREATION, SPORTS AND DANCE (GJOHPERSD)

\author{
Volume 9, Year 2016
}

A JOURNAL OF THE DEPARTMENT OF HEALTH, PHYSICAL EDUCATION AND RECREATION (HPER)

UNIVERSITY OF CAPE COAST

GHANA, WEST AFRICA 


\title{
HANDGRIP AND PINCH STRENGTH CHANGES OF TYPE II DIABETES MELLITUS PATIENTS FOLLOWING A 12- WEEK STRENGTH TRAINING PROGRAMME
}

\author{
Elvis I. Agbonlahor, Ph. D. \& Adebisi I. Hammed M. Sc. \\ Department of Human Kinetics and Sports Science, University of \\ Benin, Benin City
}

\begin{abstract}
The purpose of this study was to establish the efficacy of a 12-week strength training (ST) programme on handgrip and pinch strength in Type 2 Diabetes Mellitus (T2DM) patients in a tertiary health institution in Benin-City. This study was a pre-test, post-test control group design. A total of 36 T2DM patients participated in the study. Handgrip and pinch strength were measured using electronic hand dynamometer (in $\mathrm{kg}$ ) and mechanical pinch gauge (in $\mathrm{kg}$ ) respectively prior to and following a 12-week ST programme. Data generated were analyzed using inferential statistics of one way analysis of variance (ANOVA) and the statistical significance was accepted for $p$ value of <0.05.The findings of the study showed that ST programme had significant effects on handgrip and pinch strength of T2DM patients. It was concluded that ST programme can substantially optimize handgrip and pinch strength of patients with T2DM. Therefore, ST programme should be considered a key element in the management of T2DM patients.
\end{abstract}

Keywords: Strength training, handgrip and pinch strength, type 2 diabetes mellitus 


\section{INTRODUCTION}

Strength training (ST) provides greater strength and endurance, and is primarily used as a tool in sports and rehabilitation medicine. Muscular strength is the amount of force a muscle can produce with a single maximal effort. However, diminished grip and pinch strength can significantly limit daily activities such as carrying an objects, laundry, turning a doorknob, vacuuming, and sporting events like wrestling, tennis, football, basketball, and gymnastics (Ruprai, Tajpuriya\& Mishra, 2015). Physiologically, ST brings about enhanced muscular strength and regained loss of muscle mass in diabetes, obesity and other metabolic syndrome (Hunter, Wetzstein, Fields, Brown \&Bamman, 2000). The impairment of insulin action in major target organs such as liver and muscles is a common pathophysiological feature of T2DM. It has been observed that the activity of the mitochondrial electron transport chain is reduced in the muscles of patients with T2DM, and that muscle mitochondria are smaller in these individuals (Sreekumar\& Nair, 2007).In addition, Helmersson, Vessby, Larsson and Basu (2004) believed that diabetes causes a reduction in the number of mitochondria in the muscle cells, a decrease in glycogen synthesis and an increase in the amount of circulating systemic inflammatory cytokines, all of which have a detrimental effect on the skeletal muscles. Reliable and valid evaluation of hand strength can provide an objective index of general upper body strength. Grip strength is the result of forceful flexion of the finger joints with a maximal voluntary force that a person is able to exert under normal biokinetic conditions. Hand grip strength (HGS) is an important component to perform precise and refined fine motor activities. This is because hand functionality is considered to be vital in most of the tasks involving upper limb. HGS has long been thought of as a possible predictor of overall body strength (Smith, Smith, Martin, Henry, Weeks, \& Bryant, 2006).

The hand of human is the effector organ of the upper limb as it is capable of performing countless actions including prehension, precision, adaptation, exploration, perception and manipulation. 
Handgrip and pinch strength changes of type ii diabetes mellitus patients following a 12week strength training programme

The hand is not only a motor organ but also a very sensitive and accurate sensory receptor, which feeds back information essential for its own performance. The hand is greatly affected by diabetic musculoskeletal complication. Adequate muscle power is required for optimum productivity while decreased muscle strength is a predictor of physical limitations (Magee, 2002). Without adequate grip, pinch and forearm strength, patients with diabetes stand the risk of developing hand and forearm problems that could result in reduced performance. Often overlooked or taken for granted, the strength of one's grip plays a key role in injury prevention and overall strength development. Patients with T2DM have been reported to be more disabled in self-care tasks and other daily living activities than non-diabetic subjects because of many hand complications. The higher the gradient of the disease, the greater will be reduction of hand grip strength, agility and function (Bardan\& Lather, 2012). Furthermore, grip and pinch strength testing are commonly used to evaluate hand function for disability ratings and to assess responses to various forms of therapy. Moreover,Savas, Koroglu, Koyuncuoglu, Uzar, Celik and Tamer (2007) demonstrated that reduced grip and pinch strength was related to disability of the hands and suggested that negative influence of diabetes and obesity on muscle quality could all contribute to poor muscle function and hand weakness. It was stated by Jacquemin, Burns and Little (2004) that weakness of hand muscles is a symptom of large number of pathologies which could result in loss of hand function. Grips reflect the strength generated by the contraction of the various arm and hand muscles involved in the activity of the hand. The amount of strength generated can then be used as a quantitative measurement of the development of hand function (Mitchell, 1976).

Furthermore, Komal and Suvarna (2015) found a significant effect of ST on grip strength and hand function in diabetic patients but they did not test for pinch strength in their study. Contrarily, Anderson, Gadeberg, Brock, and Jakobsen (1997) and Andersen, Nielsen, Mogensen and Jakobsen (2004) concluded that hand grip strength is not compromised in T2DM following ST programme. It 
was also reported by Ozdirenc, Biberoglu, and Ozcan (2003) that hand grip strength value was found to be significantly lower in the diabetic group compared with the control group. Again, the key pinch strength value for the right hand was significantly lower in the diabetic group, whereas for the left hand, the value was lower than in the control group but was insignificant following few weeks of ST programme. A similar study by Cetinus, Buyukbese, Uzel, Ekerbicer and Karaoguz (2005) showed that grip strength and key pinch power values were found to be significantly lower in patients with T2DM than in age-matched control subjects following ST programme. It was concluded in a study that was conducted by Santos, Montrezol, Pauli, Sartori-Cintra, Colantonio and Pauli (2014)on undulatory physical resistance training programme in elderly type 2 diabetics that resistance training programme used with weekly differential overloads was effective and efficient in providing significant increases in maximal muscular strength, both in lower and upper limbs in T2DM individuals. Another significant study was carried out by Kwon, $\mathrm{Han}, \mathrm{Ku}, \mathrm{Koo}, \mathrm{Kim}$ and Min (2010) and they concluded that low intensity ST was effective in increasing muscle mass and strength and reducing total fat mass without change of insulin sensitivity in T2DM patients. In the same view, an observational study carried out by Shambhuvani, Diwan and Vyas (2015) on the effect of longstanding T2DM on handgrip strength showed that grip strength was significantly reduced in diabetic individuals as compared to non-diabetic individuals. However, they also observed significant difference in dominant and non dominant handgrip strength in diabetic group and non-diabetic group and thereafter submitted that the causes of lower quantitative values of hand grip strength and pinch power in patients with T2DM are unclear.

There is no doubt that improving the handgrip and pinch strength of diabetes patients through strength training programme will go a long way in improving their functional status to meet with their life challenges. Since diabetes patients' care requires a multidisciplinary approach and guidance, this study is intended to 
Handgrip and pinch strength changes of type ii diabetes mellitus patients following a 12week strength training programme

approach the diabetes patients' care through strength training programme. Furthermore, little or no known researches have been undertaken in Nigeria and in Edo state in particular, regarding handgrip and pinch strength changes through exercise therapy. This observed gap in knowledge and research efforts informed the need for the present study.

\section{Research hypotheses}

The following hypotheses were formulated and tested at the 0.05 alpha level.

1. There is no significant difference in the handgrip strength of T2DM patients prior to and following a12-week ST programme.

2. There is no significant difference in the pinch strength of T2DM patients prior to and following a12-week ST programme.

\section{MATERIAL AND METHODS}

This study was a pre-test, post-test control group experimental design of the effects of a 12-week ST programme on handgrip and pinch strength of T2DM patients. The population of this study included 54T2DM patients between the biological ages of 51 to 73 years who were receiving treatment at the Endocrinology Unit of Internal Medicine Department, University of Benin Teaching Hospital, Benin-City, Nigeria.A total of 36 patients with T2DM in the above mentioned hospital participated in this study. They were recruited using the simple random sampling technique. Balloting without replacement was used to select two-third $(2 / 3)$ of the population for the study. The names of the patients were written on pieces of paper each and these pieces of paper were put in a bag from where one piece of paper was picked at a time and the name on the piece of paper picked was recorded. This process was repeated until the desired sample size 
was obtained. Thereafter, the recorded names were also assigned randomly into two (2) groups (experimental and the control groups). Eighteen (18) participants were assigned to experimental group and the other eighteen (18) to the control group using the same process. The first name in the list was assigned to experimental group and the second name to the control group, the procedure was continued till the last name in the list was assigned.

The research instrument for this study was an adaptation of Sharkely (1990) experimental protocols.

Hand grip strength of both hands was measured using a Camry Electronic Hand Dynamometer (Model: EH101). It comes with dual scale readout of forces in kilograms and pounds and however, all readings were recorded in kilograms in the present study.Also,mechanical pinch gauge was used to measure the three basic pinch tests of both hands including key pinch (lateral pinch) thumb pad to lateral aspect of middle phalanx of index finger, palmer pinch (chuck pinch) - thumb pad to pads of the index and middle fingers, and tip pinch (thumb-index pulp pinch) - thumb tip to index fingertip. It is calibrated in pounds and kilograms of force and all readings were equally recorded in kilograms in the present study.

The test instrument was an adaptation of Sharkely (1990) experimentation. However, the test instrument was certified by experts in exercise physiology and physiotherapy as appropriate for the study. The validation was effected at the Outpatients Unit of Physiotherapy Department, University of Benin Teaching Hospital, Benin-City. The following variables were measured: the handgrip and pinch strength as well as hand function of T2DM patients. It was observed that the use of the facility and procedure were feasible for the conduct of the study. This department was equally served as the project site. Also, a pilot study was conducted to establish the suitability of using the instrument for T2DM to which eight (8) T2DM patients, four (4) per group were used. The split-half method of reliability was used in obtaining the 
Handgrip and pinch strength changes of type ii diabetes mellitus patients following a 12week strength training programme

data that were subjected to Pearson Product Coefficient of Correlation. A Coefficient of 0.79 was obtained and it was considered high reliability and therefore justified the suitability and relevance of using the instrument and protocol for the study.

The study received ethical approval from the Research Ethics Committee of the hospital to conduct this study. All the participants were recruited consecutively through their hospital files at the Endocrinology Unit of Internal Medicine Department, University of Benin Teaching Hospital, Benin-City. Prior to ST programme, a detailed explanation of the test, training programme and the objectives of the study was provided for the participants who signed informed consent form. Thereafter, the participants were randomly assigned to experimental and the control groups. The handgrip and pinch strength of both groups were measured before the training and then the participants were subjected to a 12week ST programme of a frequency of 3 times per week (Monday, Wednesday, and Friday) with each session lasted for 50 minutes at $70 \%$ one-repetition maximum $(70 \% 1 \mathrm{RM})$ consisted of 2 sets of 8 repetitions for each muscle group with 3 minutes rest between sets. Periodization of the training was based on the recommendation of progressive ST for adults and T2DM (American College of Sports Medicine position stand, 2009). In this way, the protocol consisted of a weekly alteration of the intensity divided into a week of moderate overload $(70 \%$ of $1 \mathrm{RM}, 8$ repetitions).The ST programme was purely on upper limbs muscle strengthening that was aimed at improving handgrip and pinch strength of the participants. The training programme included bench press, military press, arm curl and latissimus pull, which were performed on a multi weight-lifting machine. Thereafter, handgrip and pinch strength of the participants was equally measured after the training.

\section{Grip strength measurement}

To standardize the measurement, the following guidelines were established; the arm positioning followed the American Society of Hand Therapists guidelines (Fess, 1992), with the 
subject comfortably seated with the shoulder slightly forward and the elbow flexed at a $90^{\circ}$ angle, with the forearm and wrist in a neutral position. A demonstration of how to use the device was first given to each participant by the researcher, to familiarize the participant with the use of the apparatus and to eliminate the element of fear. Alternately, three maximum power gripping efforts were made by each hand of the participant, with threesecond contractions and ten-second rest periods between the attempts and only the best of the three attempts was recorded. Motivation, such as verbal encouragement and competition between group members was used maximally. No assistance of the hand under test was allowed, but facial grimaces and associated movements of the other hand were not discouraged. The device was adjusted for different hand sizes and preferences by adjusting the centre knob and its calibration was also assessed periodically throughout the study.

\section{Pinch strength measurements}

The following guidelines were followed in the measurements of pinch strength; the gauge was "zeroed" before each pinch test by rotating the small curled knob on top of the dial indicator in a counterclockwise direction until it rests against the black pointer at the zero marking. As in grip strength measurement, test instructions and motivation were equally provided.

Key pinch (lateral pinch): The participant comfortably seated or upright, test arm at the side with elbow flexed $90^{\circ}$, palm facing inward, pinch gauge between flexed PIP joint of index finger and thumb, the researcher stood in front of the participant to the side stabilizing the pinch gauge and then had the participant to squeeze, hold and release the pinch gauge (i.e. participant applied pinch force at the pinch groove while holding the pinch gauge between his/her thumb and index fingers). Here, as muscle fatigue begins with the first concentrated effort, a single maximum effort only was recorded. 
Handgrip and pinch strength changes of type ii diabetes mellitus patients following a 12week strength training programme

Palmer pinch (chuck pinch): The participant comfortably seated or upright, test arm at the side with elbow flexed $90^{\circ}$, palm facing downward, pinch gauge between thumb and the index and middle fingers, the researcher's position and duty were the same as in key pinch measurement and also a single maximum effort was recorded.

Tip pinch (thumb-index pulp pinch): Here, the measurement protocol is the same as in palmer pinch (chuck pinch) except that the pinch gauge was between thumb and test finger without interference of other fingers.

\section{Method of Data Analysis}

An inferential statistics of one-way analysis of variance (ANOVA) was used to test the hypothesis. Then, Turkey's honesty significant difference post-hoc test was used to identify the source of the difference between the groups. Statistical significance was accepted for $p$ value of $<0.05$

\section{Results}

The results are presented in Tables $1-4$.

Table 1: Analysis Of Variance (ANOVA) Showing Difference in the Handgrip Strength of the Participants

\begin{tabular}{lrrrcr}
\hline & Sum of Squares & df & Mean Square & F & Sig. \\
\hline Between Groups & 108.365 & 3 & 36.122 & 3.420 & .022 \\
Within Groups & 718.221 & 68 & 10.562 & & \\
Total & 826.586 & 71 & & & \\
\hline
\end{tabular}

Df-degree of freedom, F-test is a ratio of sample variance, Sig.-the two-tailed p-value associated with the null that the groups have the same variance.

From Table 1, difference in the handgrip strength prior to and following a 12-week ST programme was determined using one way analysis of variance (ANOVA). The F-value of 3.420 with 3 and 71 degree of freedom was found to be statistically significant 
at $0.05(p<0.05)$. Thus, the hypothesis which stated that therewould be no significant difference in the handgrip strength of T2DM patients prior to and following a12-week ST programme was rejected. It therefore implies that ST had substantial effect on the handgrip strength ofT2DM patients. This however, necessitated probing into the post-hoc test to identify the source of the significance.

Table 2: Turkey's Honesty Significant Difference Post Hoc Test Showing Difference in the Handgrip Strength of the Participants

\begin{tabular}{llrrr}
\hline (I) Group & (J) Group & $\begin{array}{r}\text { Mean Difference } \\
(\mathrm{I}-\mathrm{J})\end{array}$ & $\begin{array}{c}\text { Std. } \\
\text { Error }\end{array}$ & Sig. \\
\hline \multirow{3}{*}{ Pre-exp } & Post-exp & $-3.11667^{*}$ & 1.08331 & .027 \\
& Pre-control & -.45000 & 1.08331 & .976 \\
& Post-control & -.51111 & 1.08331 & .965 \\
Post-exp & Pre-exp & $3.11667^{*}$ & 1.08331 & .027 \\
& Pre-control & 2.66667 & 1.08331 & .075 \\
& Post-control & 2.60556 & 1.08331 & .086 \\
Pre-control & Pre-exp & .45000 & 1.08331 & .976 \\
& Post-exp & -2.66667 & 1.08331 & .075 \\
& Post-control & -.06111 & 1.08331 & 1.000 \\
Post-control & Pre-exp & .51111 & 1.08331 & .965 \\
& Post-exp & -2.60556 & 1.08331 & .086 \\
& Pre-control & .06111 & 1.08331 & 1.000 \\
\hline
\end{tabular}

Turkey's honesty significant difference test was carried out to determine the difference in variation in the hand grip strength of the participants. However, all the pair wise of mean difference were found to be statistically insignificant $(p>0.05)$ except pre-exp versus post-exp $\left(-3.11667^{*}\right)$ as shown in Table 2 . This implies that the entire pair wise mean had variation. Therefore, the treatment influenced the variation of the handgrip strength of the participants. 
Handgrip and pinch strength changes of type ii diabetes mellitus patients following a 12week strength training programme

Table 3: Analysis Of Variance (ANOVA) Showing Difference in the Pinch Strength of the Participants

\begin{tabular}{lrrrrr}
\hline & Sum of Squares & df & Mean Square & F & Sig. \\
\hline Between Groups & 187.330 & 3 & 62.443 & 12.017 & .000 \\
Within Groups & 353.348 & 68 & 5.196 & & \\
Total & 540.678 & 71 & & & \\
\hline
\end{tabular}

Df-degree of freedom, F-test is a ratio of sample variance, Sig.-the two-tailed p-value associated with the null that the groups have the same variance.

From Table 3, difference in the pinch strength prior to and following a 12-week ST programme was determined using one way analysis of variance (ANOVA). The F-value of 12.017 with 3 and 71 degree of freedom was found to be statistically significant at $0.05(p<0.05)$. Thus, the hypothesis which stated that there would be no significant difference in the pinch strength of T2DM patients prior to and following a12-week ST programme was rejected. It therefore implies that ST had substantial effect on the pinch strength ofT2DM patients, hence the probe into the post-hoc test to identify the source of the significance presented in Table 4.

Table 4: Turkey's Honesty Significant Difference Post Hoc Test Showing Difference in the Pinch Strength of the Participants

\begin{tabular}{llrrr}
\hline (I) Group & (J) Group & $\begin{array}{c}\text { Mean Difference } \\
\text { (I-J) }\end{array}$ & $\begin{array}{c}\text { Std. } \\
\text { Error }\end{array}$ & Sig. \\
\hline \multirow{3}{*}{ Pre-exp } & Post-exp & $-4.08333^{*}$ & .75985 & .000 \\
& Pre-control & -.60556 & .75985 & .856 \\
& Post-control & -.60000 & .75985 & .859 \\
Post-exp & Pre-exp & $4.08333^{*}$ & .75985 & .000 \\
& Pre-control & $3.47778^{*}$ & .75985 & .000 \\
& Post-control & $3.48333^{*}$ & .75985 & .000 \\
Pre-control & Pre-exp & .60556 & .75985 & .856 \\
& Post-exp & $-3.47778^{*}$ & .75985 & .000 \\
Post-control & Post-control & .00556 & .75985 & 1.000 \\
& Pre-exp & .60000 & .75985 & .859
\end{tabular}


Elvis I. Agbonlahor, Ph. D. \& Adebisi I. Hammed M. Sc.

Post-exp

$\begin{array}{lll}-3.48333^{*} & .75985 & .000\end{array}$

Pre-control $\begin{array}{lll}-.00556 & .75985 & 1.000\end{array}$

Turkey's honesty significant difference test was carried out to determine the difference in variation in the pinch strength of the participants. However, all the pair wise of mean difference were found to be statistically insignificant $(p>0.05)$ except pre-exp versus post-exp $\left(-4.08333^{*}\right)$, post-exp versus pre-control $\left(3.47778^{*}\right)$, post-exp versus post-control $\left(3.48333^{*}\right)$, pre-control versus post-exp $\left(-3.47778^{*}\right)$ and post-control versus post-exp ($\left.3.48333^{*}\right)$ as shown in Table 4 . This implies that the entire pair wise mean had variation. Therefore, the treatment influenced the variation of the handgrip strength of the participants.

\section{Discussion}

This study showed that the ST programme administered had substantive impact on the participants' handgrip strength. This finding is in agreement with Komal and Suvarna (2015); Sheri, Ronald, Bo, Judith, Bryan, Richard and Barry (2010); Bacchi, Negri, Targher, Faccioli and Lanza (2013);Kwon, Han, Ku, Koo, Kim and Min (2010)and Chou, Hwang and Wu (2012) submissions thatST programme had significant effect on handgrip strength in T2DM patients. It was also concluded in the study that was conducted by Santos, Montrezol, Pauli, Sartori-Cintra, Colantonio and Pauli (2014)on undulatory physical resistance training programme in elderly type 2 diabetics that resistance training programme used with weekly differential overloads was effective and efficient in providing significant increases in maximal muscular strength, both in lower and upper limbs in T2DM individuals. In contrast, Anderson, Gadeberg, Brock, and Jakobsen (1997) and Andersen, Nielsen, Mogensen and Jakobsen (2004) concluded that hand grip strength is not compromised in T2DM following ST programme. These contrasting findings might not be unconnected to variation in study methodology including subject 
Handgrip and pinch strength changes of type ii diabetes mellitus patients following a 12week strength training programme

characteristics or differences in measuring instruments of handgrip strength.

Moreover, the significant effect of ST programme on handgrip strength found in this study can probably be explained by its effects on the activity of the mitochondrial electron transport chain via increasing the size of mitochondria which is ordinarily smaller in the muscles of patients with T2DM. It could also be that the ST programme brought about quality changes in the muscles and increases muscle fibres' cross-sectional area or causea higher proportion of type IIA fibres at the expense of type IIB fibres, in other words a change towards higher oxidative capacity and slower contraction speed which could enable sustained handgrip strength. In addition, the ability of ST programme to combat a reduction in the number of mitochondria in the muscle cells, a decrease in glycogen synthesis and an increase in the amount of circulating systemic inflammatory cytokines which all have a detrimental effect on handgrip strength of T2DM patients may be responsible for a significant increase in handgrip strength observed in these individuals.Similarly, the present study also revealed that the ST programme administered had significant effects on pinch strength of the participants. This finding is supported by the study of Komal and Suvarna (2015). On the contrary, the study is not in agreement with the studies of Ozdirenc, Biberoglu, and Ozcan (2003) and Cetinus, Buyukbese, Uzel, Ekerbicer and Karaoguz (2005). These contrasting findings can be viewed in two different perspectives. Firstly, differences in the gradients or clinical characteristics of T2DM morbidity as the root of initial episode of variation. Secondly, the ST programme administeredimpacted strongly on both the intrinsic and extrinsic muscles of the hand which couldexplain the better pinch strength gain observed in the present study.However, the limitations of this study were that only the ST was considered because of difficulty in monitoring other activities of the participants, such as prescribed medications and other home programmes and, the severity of T2DM was not taken into consideration due to inadequate clinical investigations and porosity in documentation. 


\section{CONCLUSION}

This study therefore concluded that the ST programme can substantially optimize handgrip and pinch strength gains of patients with T2DM. Therefore, the ST programme is a good training modality for improving handgrip and pinch strength of patients with T2DM.

Based on the findings, the following recommendations were made:

1. ST programme should be considered a key element in the management of T2DM.

2. Handgrip and pinch strength measures should be introduced into clinical practice.

\section{REFERENCES}

Andersen, H., Nielsen, S., Mogensen, C.E., \&Jakobsen, J. (2004). Muscle strength in type 2 diabetes. Diabetes, 53, $1543-1548$.

Anderson, H., Gadeberg, P.C., Brock, B., \&Jakobsen, J. (1997). Muscular atrophy in diabetic neuropathy: A stereological magnetic resonance imaging study. Diabetologia, 40, 1062-1066.

Bacchi, E., Negri, C., Targher, G., Faccioli, N. \&Lanza, M. (2013). Both resistance training and aerobic training reduce hepatic fat content in type 2 diabetic subjects with nonalcoholic fatty liver disease (the RAED2 randomized trial). Hepatology, 58, 1287-1295.

Bardan, M. \& Lather I. (2012). Type 2 diabetes mellitus in Arabic speaking countries. Int. J. Endocrinol, 9, 28-73.

Centinus, E., Buyukbese, M.A., Uzel, M., Ekerbicer, H. \&Karaoguz, A. (2005).Handgrip strength in patients 
Handgrip and pinch strength changes of type ii diabetes mellitus patients following a 12week strength training programme

with type 2 diabetes mellitus.Diabetes Research and Clinical Practice, 3346, 1-9.

Helmersson, J., Vessby,B., Larsson,A. \&Basu,S. (2004). Association of type 2 diabeteswith

cyclooxygenase-mediated inflammation and oxidative stress in an elderly population.

Circulation, 109, $1729-1734$.

Hunter, G.R., Wetzstein, C.J., Fields, D.A., Brown, A., \&Bamman, M.M. (2000). Resistance training increases total energy expenditure and free-living physical activity in older adults. Journal of Applied Physiology, 89, 977-84.

Jacquemin, G.L., Burns, S.P. \& Little, J.W. (2004). Measuring hand intrinsic muscles strength: A normal values and interrater reliability. Journal of Spinal cord Medicine, 27, 460-7.

Komal, D.T., Suvarna, G. (2015). Effectiveness of strength training on hand functions in patients with diabetic heomopathy, Indian Journal of Basic and Applied Medical Research, 4, 429-437.

Kwon,H.R., Han,K.A., Ku,Y.H., Ahn, H.J., Koo,B.K., Kim,H.C. \&Min,K. W. (2010). The effects of resistance training on muscle and body fat mass and muscle strength in type 2 diabetic women. Korean Diabetes J, 34, 101-110.

Magee, D. (2002). Orthopedic Physical Assessment (4th ed.). Penny sylovania, Elsevier science, 355-418.

Mitchell, J. (1976). A measurement of hand function in the normal child and the cerebral palsied child. Australia Journal of Physiotherapy, 22(4), 161-165.

Ozdirenc, M., Biberoglu, S. \&Ozcan, A. (2003).Evaluation of physical fitness in patients with type 2 diabetes mellitus. Diabetes Research and Clinical Practice, 60(3), 171-176.

Ruprai, R.K, Tajpuriya, S.V. \& Mishra, N. (2015). Handgrip strength as determinant of upper body strength/physical fitness: a comparative study among individuals performing 
gymnastics (ring athletes) and gymnasium (power lifters).International Journal of Medical Science and Public Health, 5, 1- 6.

Santos, G.M., Montrezol, F.T., Pauli, L.S., Sartori-Cintra, A.R., Colantonio, E., Gomes, R.J., Marinho, R., Moura, L.P., Pauli, J.R.(2014).Undulatory physical resistance training program increases maximal strength in elderly type 2 diabetics.Einstein, 12(4):42532.

Savas, S., Koroglu, B.K., Koyuncuoglu, H.R., Uzar, E., Celik, H. \& Tamer, N.M. (2007). The effects of diabetic related soft tissue hand lessions and the reduced hand strength on functional disability of hand in type 2 diabetic patients. Diabetes Research and Clinical Practice, 77(1), 77-83.

Shambhuvani, M.C., Diwan, S.J. \&Vyas, N.J. (2015). Effect of longstanding diabetes mellitus II on handgrip strength: An observational study. IAIM, 2(5), $\quad$ 135139.

Sheri, R.C., Ronald, J.S., Bo, F., Judith, G. R., Bryan, J. B., Richard, R. R. \& Barry, B. (2010). Exercise and type 2 diabetes: The American College of Sports Medicine and the American Diabetes Association joint position statement. Diabetes Care, 33(12), 147-67.

Smith, T., Martin, M., Henry, R. Weeks, S. \& Bryant, A. (2006). Grip Strength in Relation to Overall Strength and Functional Capacity in Very Old and Oldest Old Females. The Haworth Press Inc., 63-78. 